\title{
Monitoring Behaviorally Induced Biochemical Changes Using Fluorescence Lifetime Photometry
}

\author{
Suk Joon Lee', Yao Chen ${ }^{1,2}$, Bart Lodder ${ }^{1,3}$ and Bernardo L. Sabatini ${ }^{1 *}$ \\ ${ }^{1}$ Howard Hughes Medical Institute, Department of Neurobiology, Harvard Medical School, Boston, MA, United States, \\ ${ }^{2}$ Department of Neuroscience, Washington University School of Medicine, St. Louis, MO, United States, ${ }^{3}$ Master Program \\ Neuroscience and Cognition, Graduate School of Life Sciences, Utrecht University and Department of Translational \\ Neuroscience, Brain Center, University Medical Center, Utrecht, Netherlands
}

OPEN ACCESS

Edited by: Keith Mathieson, University of Strathclyde, United Kingdom

Reviewed by:

Guohong Cui,

National Institutes of Health $(\mathrm{NIH})$,

United States

Minmin Luo,

Tsinghua University, China

*Correspondence:

Bernardo L. Sabatini

bsabatini@hms.harvard.edu

Specialty section:

This article was submitted to

Neural Technology,

a section of the journal

Frontiers in Neuroscience

Received: 06 June 2019

Accepted: 09 July 2019

Published: 31 July 2019

Citation:

Lee SJ, Chen Y, Lodder B and

Sabatini BL (2019) Monitoring

Behaviorally Induced Biochemical

Changes Using Fluorescence Lifetime

Photometry. Front. Neurosci. 13:766.

doi: 10.3389/fnins.2019.00766
All cells respond to extracellular signals by altering their intracellular biochemical state. In neurons, such signaling regulates many aspects of cell and synapse biology and induces changes that are thought to be important for nervous system development, its adaptation in the face of a changing environment, and ongoing homeostatic maintenance. Although great advances have been made in developing novel fluorescent reporters of intracellular signaling as well as in methods of fluorescence detection for use in freely moving animals, these approaches have generally not been combined. Thus, we know relatively little about how the intracellular biochemical state of neurons, and other cell classes, is dynamically regulated during animals' behavior. Here we describe a single multi-mode fiber based fluorescence lifetime photometry system (FLiP) designed to monitor the state of fluorescence reporters of biochemical state in freely moving animals. We demonstrate the utility of FLiP by monitoring the lifetime of FLIM-AKAR, a genetically encoded fluorescent reporter of PKA phosphorylation, in populations of direct and indirect pathway striatal projection neurons in mice receiving food rewards. We find that the activity of PKA in each pathway is transiently regulated by reward acquisition, with PKA phosphorylation being enhanced and repressed in direct and indirect pathway neurons, respectively. This study demonstrates the power of FLiP to detect changes in biochemical state induced by naturalistic experiences in behaving animals.

Keywords: FLIM (fluorescence lifetime imaging microscopy), fiber photometry, PKA, dopamine, accumbens

\section{INTRODUCTION}

Neurons in the mammalian brain utilize transmembrane signaling to regulate their intracellular state in response to extracellular cues. One prominent mode of such regulation is through the activation of G-protein coupled receptors, which transduce the binding of an extracellular ligand to liberate effector G-proteins and generate second messengers (Greengard, 2001; Kreitzer and Malenka, 2008; Chen et al., 2017). Similarly, Ca-permeable ion channels, such as voltage-gated Ca channels and NMDA-type glutamate receptors, increase intracellular calcium and activate Cadependent signaling cascades (Lee et al., 2009; Herring and Nicoll, 2016). Other extracellular signals, including neurotrophins, cytokines, and gasses also rapidly induce changes in cellular biochemical state (Huang and Reichardt, 2001; Regehr et al., 2009; Prieto and Cotman, 2017). 
Such modulation of intracellular signaling by extracellular signals is central to activity-dependent signaling cascades, including those that mediate synaptic plasticity, dendritic protein translation, the regulation of kinases and phosphatases, and genetranscription (Nayak et al., 1998; Carvalho et al., 1999; Shaywitz and Greenberg, 1999; Lau et al., 2004; Skeberdis et al., 2006; Lee et al., 2009; Kandel, 2012; Yagishita et al., 2014). Thus, these pathways are thought to be crucial for activity-dependent circuit formation and refinement during development as well as for learning, memory formation, and behavioral adaptation in response to a changing environment (Kandel and Abel, 1995; Davis, 1996; Beninger and Gerdjikov, 2004; Tronson et al., 2006; Doll and Broadie, 2014; Jiang and Nardelli, 2016). To understand how these pathways are activated and how they contribute to these functions, one would ideally monitor the dynamics of the upstream activating second messenger (such as Ca or cAMP), the specific activity of effector enzyme itself (such as the activity state of a kinase), and the state of the enzyme substrates (such as the phosphorylation state of a kinase target).

Protein kinase A (PKA) in the striatum has been proposed to be an essential mediator of dopamine dependent rewardreinforced behaviors in animals (Beninger and Gerdjikov, 2004; Kravitz and Kreitzer, 2012). The dorsal striatum and the ventral striatum nucleus accumbens (NAc) receive dense innervation from dopaminergic neurons located in the substantia nigra pars compacta (SNC) and ventral tegmental area (VTA), respectively (Howe and Dombeck, 2016). Dopamine release in the striatum is generally triggered by positive action outcomes, such as the acquisition of water or food, and, through processes that are still poorly understood, modulates striatal circuits to promote the repetition of the action that led to the good outcome (Schultz et al., 1997; Bromberg-Martin et al., 2010; Schultz, 2013). Central to models of dopamine action on the striatum and reward reinforcement are its differential effects on the two main classes of striatal projection neurons (SPNs) (Berke and Hyman, 2000; Shen et al., 2008; Bromberg-Martin et al., 2010; Kravitz and Kreitzer, 2012). These models propose that dopamine acting through $\mathrm{G}_{\alpha s}$-coupled Type 1 dopamine receptors (D1Rs) expressed by direct pathway SPNs (dSPNs) promotes motor action and reward reinforcement by activating PKA in these cells to increase their intrinsic excitability, potentiate synapses, and induce transcription (Gerfen and Surmeier, 2011; Tritsch and Sabatini, 2012). Conversely, dopamine acting through $\mathrm{G}_{\alpha i^{-}}$ coupled Type 2 dopamine receptors (D2Rs) expressed by indirect pathway SPNs (iSPNs) may induce opposite effects in these cells (Gerfen and Surmeier, 2011; Tritsch and Sabatini, 2012). These push-pull actions of dopamine are thought to induce imbalances in activities and biochemical states of dSPNs and iSPNs that contribute to reward reinforced learning, drug addiction, and Parkinson's disease (Bromberg-Martin et al., 2010; Kravitz and Kreitzer, 2012). However, due to technical limitations (Goto et al., 2015; Yamaguchi et al., 2015), the dynamic change of PKA activity in dSPNs and iSPNs during behavior is unclear, and thus basic tenets of this model remain untested.

Here, we developed a system to monitor PKA signaling in genetically-defined neurons, such as dSPNs and iSPNs, in freely behaving mice. We exploit a genetically encoded sensor for
PKA, FLIM-AKAR (Chen et al., 2014), which contains a GFPbased fluorophore whose lifetime in the excited state changes when the reporter is phosphorylated by PKA. In order to use this sensor in freely moving mice, we designed a single optical fiber system for fluorophore lifetime measurements, building on the previous work that used a pair of optical fibers to monitor the static lifetime of a genetically encoded fluorophore in vivo (Cui et al., 2013, 2014). We refer to this approach as Fluorescence Lifetime Photometry (FLiP). We validated this approach by performing bulk measurements of fluorophore lifetime change in vivo and demonstrate the ability of FLiP to detect dynamic modulation of PKA activity in the NAc. Our results demonstrate that environmental signals, such as the acquisition and consumption of food, trigger brief and opposing PKA phosphorylation transients in dSPNs and iSPNs. We propose that FLiP can be used with other fluorescence lifetime reporters of biochemical pathways and is a powerful tool for monitoring intracellular biochemical states in vivo.

\section{RESULTS}

\section{Analysis of Fluorescein Standards Using 2P-FLIM and FLiP}

A previous study by Cui et al. (2013) measured the fluorescence lifetime of a fluorophore in the brain of a mouse using a dual fiber photometry system in which a single-mode fiber delivered excitation light into the brain and a multi-mode fiber collected light in the brain. The rationale behind this design was that a single-mode fiber provides more stable illumination than a multimode fiber, making it more suitable for excitation. Conversely, a multi-mode fiber has higher light collection efficiency than a single-mode fiber, making it more suitable for fluorescence collection. Since it is difficult to launch a free space laser into a single mode fiber, and two fibers are bulky and more damaging when inserted into the brain, we examined if bulk fluorescence lifetime measurements could be made through a single multimode fiber. In addition, we examined if the sensitivity of such a system was sufficient to detect small changes in fluorescence lifetime of a FÖrster resonance energy transfer (FRET)-based reporter of an intracellular biochemical signal in freely moving animals.

To test these possibilities, we built a FLiP system that relays pulsed excitation light to an optical fiber and detects fluorescence emission by time correlated single photon counting (Figure 1). The FLiP setup was versatile and could be used with either a pair of multi-mode optical fibers for independent excitation and emission paths or with a single multi-mode optical fiber for both excitation and emission light. We used the setup to measure the lifetime of fluorescein in a standard solution and compared the measurements to those made by a conventional 2-photon fluorescence lifetime imaging microscope (2P-FLIM) (Figure 2A). On average, the lifetime of a fluorescein standard was underestimated by FLiP (3.4-3.8 ns) compared to with $2 \mathrm{P}$ FLIM (4.1 ns) (Figures 2B,C). This deviation likely arises from the contribution of fiber autofluorescence, which has a shorter lifetime $[2.7 \pm 0.1 \mathrm{~ns}(\mathrm{SD}), n=6$, measured by launching laser 


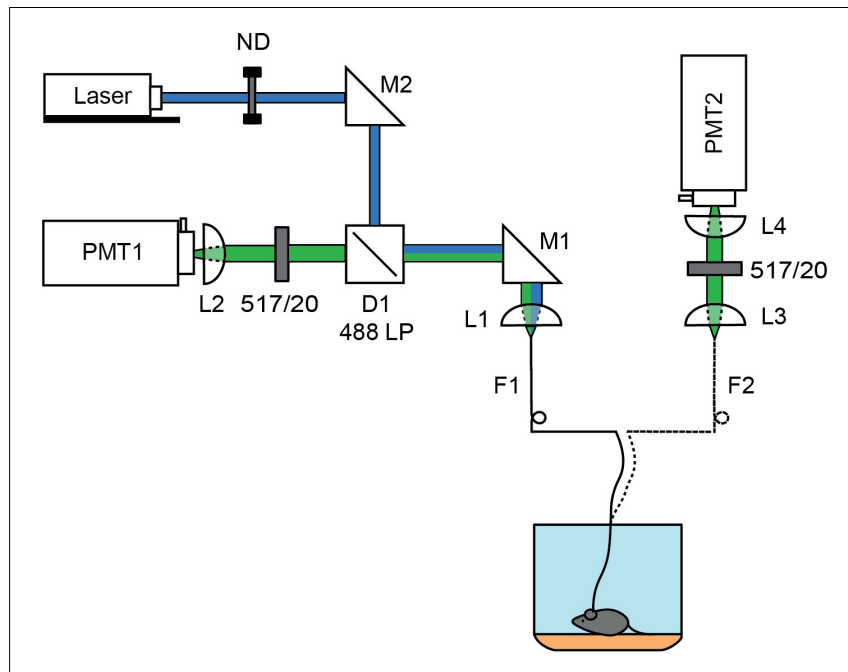

FIGURE 1 | Schematics of 1 and 2 fiber fluorescence lifetime photometry system. A $473 \mathrm{~nm}$ pulsed laser (blue) was attenuated using a rotating neutral density filter (ND) and focused into a fiber optic (F1) patch cord using steerable mirror M1 and lens L1. For the 1-fiber based method, fluorescence was collected through the same fiber ( $F 1)$ using lens L1. Emission light was separated from the excitation light using dichroic D1 (488 LP) and a $517 / 20 \mathrm{~nm}$ emission filter before focusing the fiber face on a photomultiplier tube (PMT1) by L2. The patch cord was connected to the single fiber optic implant inserted into the brain. For the 2 -fiber based method, a dual fiber optic implant was used and connected to F1, which was used solely for excitation, and to a second fiber optic (F2) patch cord used to collect fluorescence. The F2 fiber face was imaged onto a PMT (PMT2) using lens L3 and L4 which were separated by a 517/20 nm emission filter. In each case, the PMT was connected to a time correlated single photon counting board. through the fiber into an optical dump] than fluorescein. It is also possible that because 2P-FLIM excitation light is focused onto a very small volume of fluorescein solution, it may quickly bleach fluorophore molecules and effectively prevent homoFRET. Since the fiber-based methods distribute excitation light to a large volume of fluorescein solution, they may suffer more from autoquenching.

We measured lifetimes with FLiP with optical fibers directly inserted into the fluorescein solution or with an implant that would normally be placed in the brain (Figures 2B,C, "w/ implant" vs. "w/o implant"). For both the single and dual fiber systems, the addition of the implant lowered the lifetime further, likely reflecting additional autofluorescence of the fiber and the glue in the fiber optic implant.

These measurements revealed that the single fiber setup with a fiber implant reported a fluorescein lifetime measurement closer to the 2P FLIM value than the double fiber setup with a fiber implant, suggesting that the double fiber system does not provide a significant advantage over a single fiber system. Overall, fluorescein lifetime measurements demonstrate that a single multi-mode fiber-based lifetime measurement can measure a lifetime close to a true value in a stable manner [3.55 \pm 0.02 ns (SD) of fiber measurements vs. $4.13 \pm 0.06$ ns of 2 P FLIM measurements, $n=6$ ]. Nevertheless, measuring the absolute lifetime of the targeted fluorophore may be more challenging with FLiP than with 2P-FLIM because of the contribution of fiber autofluorescence as well as the larger contribution of tissue autofluorescence to FLiP measurements (compared to that from a small excitation volume in 2P-FLIM). However, this is not a limitation for many studies in which changes in fluorescence lifetime will be used to measure dynamic engagements of a biochemical pathway in vivo.

\section{FLiP Can Detect Dopamine Receptor Driven Activation of dSPN PKA Activity in vivo}

To test if single fiber FLiP can detect a biologically meaningful signal, we used it to measure the lifetime of a PKA activity reporter (FLIM-AKAR) that we previously developed (Chen et al., 2014). Briefly, FLIM-AKAR is a FRET-based PKA activity reporter consisting of a donor fluorophore and a
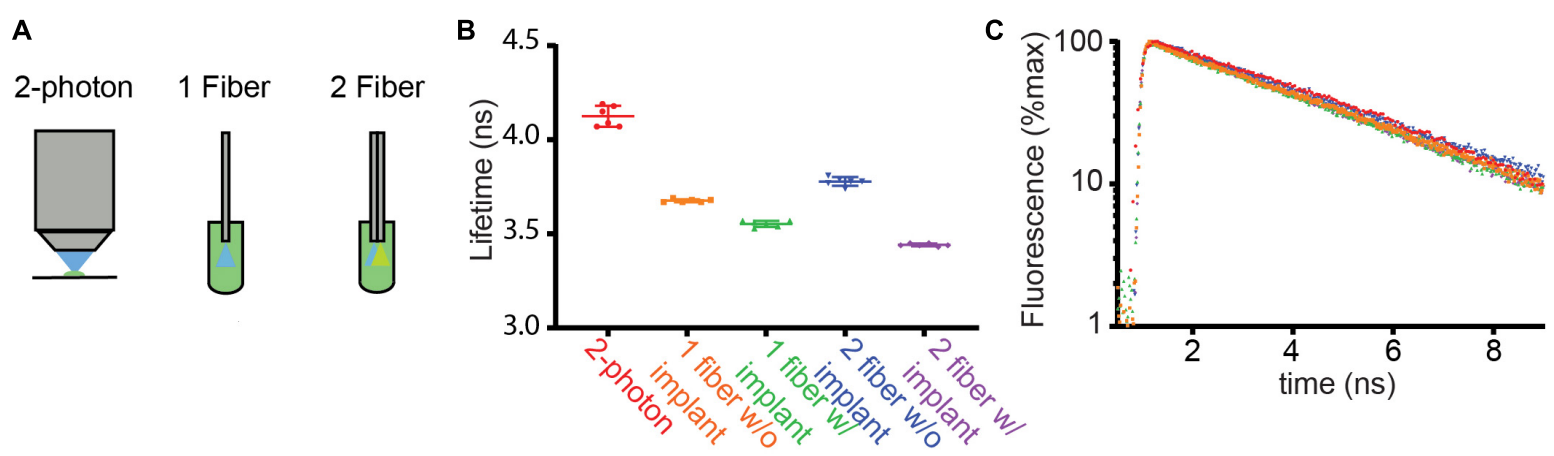

FIGURE 2 | Comparison of fluorescence lifetimes across measurement modalities. (A) Schematics of methods used to measure the fluorescence lifetime of fluorescein in solution (4 $\mathrm{\mu g} / \mathrm{ml})$. Lifetime was measured from a droplet under the objective of a 2 photon fluorescence lifetime imaging microscope (2-photon) using pulsed $920 \mathrm{~nm}$ excitation. In addition, the fiber optic based setups described in Figure $\mathbf{1}$ were used to measure lifetime with the fiber ends dipped into the fluorescein solution. (B) Average fluorescence lifetimes measured with the arrangements schematized in (A). The 1- and 2-fiber measurements were made with and without the patch cord connected to the corresponding implants that are normally inserted into the brain. The results of each of 6 measurements (dots) as well as the averages and SDs are shown (lines). (C) Example fluorescence lifetime histograms for each recording color-coded as in (B). 
dark acceptor. When the conformation of the sensor changes due to its phosphorylation by PKA, it brings the donor and the acceptor closer together, enhancing intramolecular FRET and reducing the fluorescence lifetime of the donor. We have shown that, when monitored by 2P-FLIM, FLIMAKAR fluorescence lifetime decreases upon PKA activation and increases upon PKA inactivation in neurons in brain slices (Chen et al., 2014).

We expressed FLIM-AKAR in dSPNs in the NAc by injecting an adeno-associated virus (AAV) that expressed the sensor in a
Cre-dependent manner (AAV-FLEX-FLIM-AKAR) into Drd1aCre mice and implanted a single optical fiber into the NAc (Figure 3A). After 2 weeks of expression of the reporter virus, we injected a Type 1 dopamine receptor (D1R) agonist (SKF 81297 hydrobromide, $10 \mathrm{mg} / \mathrm{kg}$ IP) and measured the lifetime change in the fluorescence signal collected from the NAc in freely moving animals. The fluorescence lifetime of FLIM-AKAR showed a significant reduction $(\sim 0.15 \mathrm{~ns})$ suggesting an increase in PKA activity in dSPNs after D1R agonist injection (Figure 3B). The magnitude of the lifetime change was comparable to that
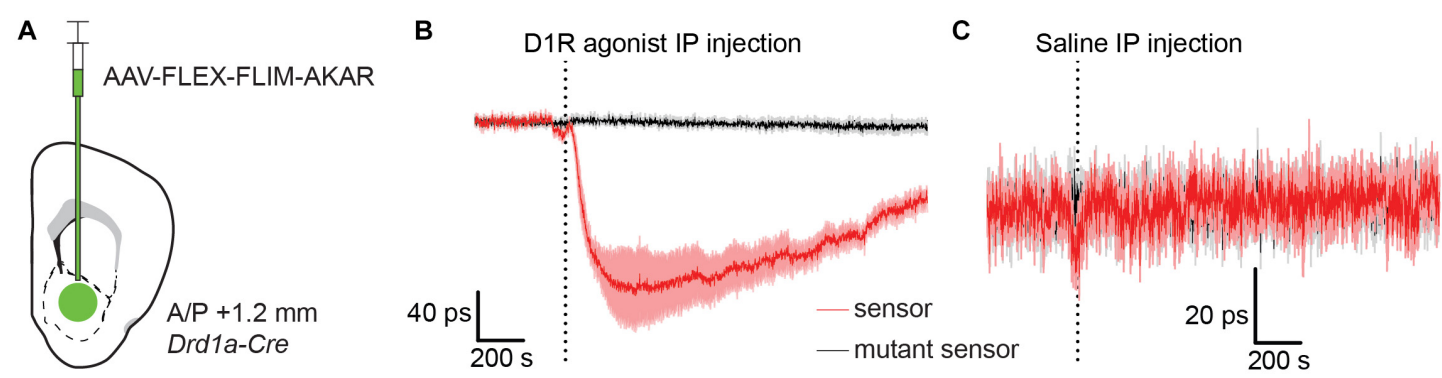

D
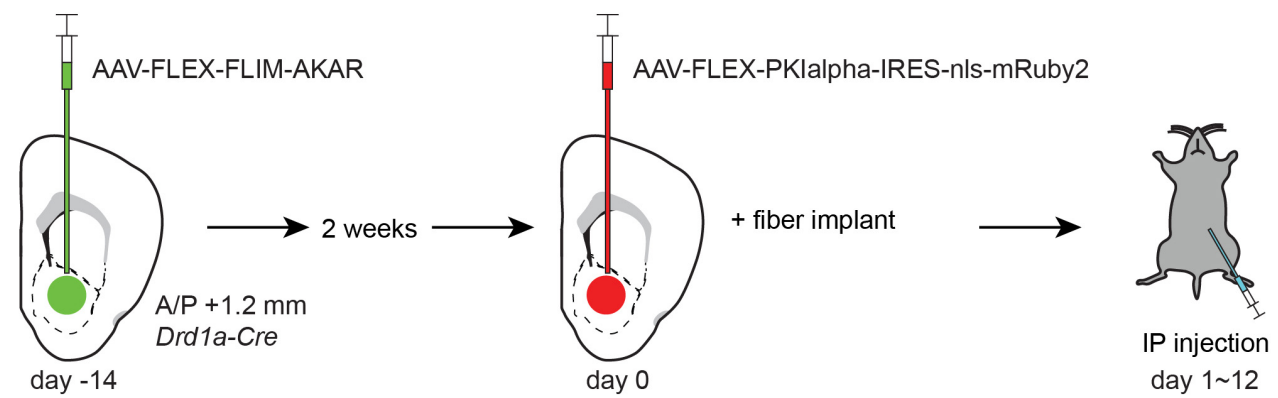

day -14

day 0

day $1 \sim 12$

E
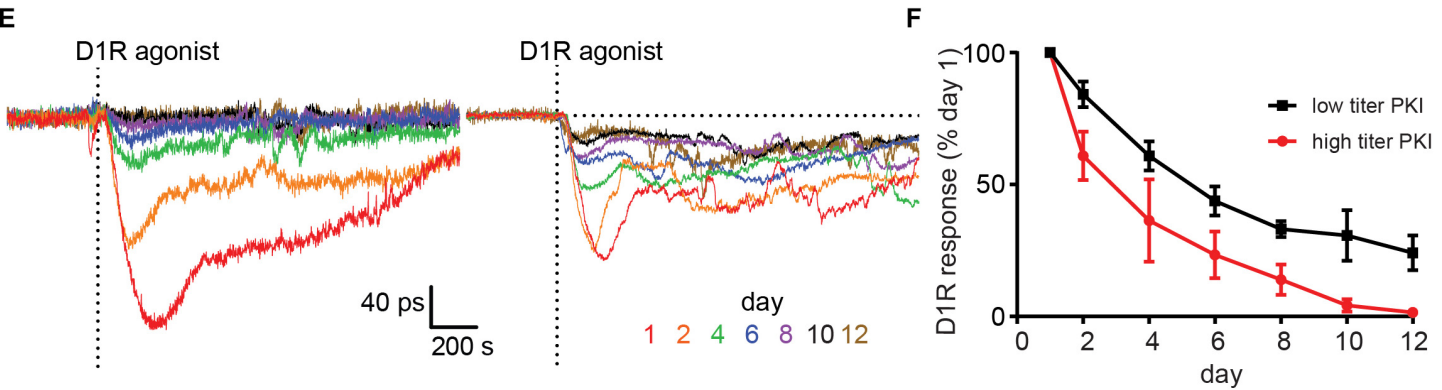

FIGURE 3 | Type 1 dopamine receptor agonists increase PKA phosphorylation in NAc dSPNs. (A) Schematic of a coronal section at $1.2 \mathrm{~mm}$ depicting viral injection of AAV-FLEX-FLIM-AKAR into the NAc of a Drd1a-Cre mouse. An optical fiber was implanted $200 \mu \mathrm{m}$ above the injection site. (B) Changes in fluorescence lifetime of FLIM-AKAR expressed in dSPNs of a Drd1a-Cre mouse that was intraperitoneally (IP) injected with a Type 1 dopamine receptor (D1R) agonist. The changes over time in fluorescence lifetime measured in 1-s interval relative to the baseline (pre-injection) period are plotted. The time when the injection finished is indicated by the dashed vertical line. For FLIM-AKAR (red), a decrease in lifetime represents an increase in phosphorylation by PKA. A similar measurement was made using FLIM-AKAR ${ }^{\text {T391A }}$ which has a point mutation at the PKA phosphorylation site (black). For the sensor and mutant sensor, the average responses (line) and SEM (shaded regions) across seven mice are shown. (C) As in (B), the responses of FLIM-AKAR (red) and the phosphorylation site mutant FLIM-AKAR ${ }^{T 391 A}$ (black) to IP injection of saline. For each, data collected across four mice are shown. (D) Experimental design for using virally-transduced PKI to validate the specificity of the FLIM-AKAR lifetime changes in dSPNs following D1R agonist injection. A Drd1a-Cre mouse was first injected with AAV-FLEX-FLIM-AKAR to express the reporter in dSPNs (left). After 2 weeks had elapsed to achieve strong expression of the reporter, the same mouse and region were injected with AAV-FLEX-PKlalpha-IRES-nlsmRuby2 (middle). An optical fiber was implanted during the same surgery used for the second viral injection. The responses to IP D1R agonist injection were measured (right) over the subsequent 1-12 days. (E) D1R agonist induced changes in FLIM-AKAR lifetime across multiple days from example mice injected with high (left) or low (1/10 th of high, right) titer PKI virus. (F) The amplitudes of D1R agonist induced changes in FLIM-AKAR lifetime relative to that on day 1 across multiple days for high (red, $n=3$ mice) and low (black, $n=4$ mice) titer PKI virus groups. Error bar $=$ SEM. 
observed previously in brain slices with activation of PKA (Chen et al., 2014).

The D1R-agonist induced fluorescence lifetime change did not occur in mice expressing a mutant reporter (FLIM-AKAR ${ }^{\text {T391A }}$ ) in dSPNs, which has a point mutation at its consensus PKA phosphorylation site (Figure 3B). This demonstrates that the phosphorylation of the sensor at this site is required for the observed lifetime change. Furthermore, IP injection of saline did not produce a comparable transient, suggesting that the lifetime change in D1R agonist experiments is due to the effect of the drug rather than a general stress caused by scruffing and injection (Figure 3C). Interestingly, there was a small but significant change in FLIM-AKAR lifetime at the time of D1R agonist or saline IP injection that was much smaller and shorter-lived than the long-lived D1R agonist response. This small signal was not seen in FLIM-AKAR ${ }^{\text {T391A }}$ expressing mice suggesting that there is a general effect of IP injection that increases dSPN PKA activity.

To further validate the specificity of the reporter fluorescence lifetime change in vivo, we employed PKI, which is one of the most specific inhibitors of PKA currently available (Dalton and Dewey, 2006; June, 2012). We had previously developed an AAV that expresses PKI in a Cre-dependent manner (Chen et al., 2014), allowing specific antagonism of PKA in a geneticallydefined population. We first injected AAV-FLEX-FLIM-AKAR into the NAc of a Drd1a-Cre mouse and waited 2 weeks to achieve stable expression of the reporter (Figure 3D). We subsequently injected AAV-FLEX-PKIalpha-IRES-nls-mRuby2 into the same location and performed fiber implantation. This approach allowed us to measure PKA dynamics in dSPNs as PKI expression increased starting the day after fiber implantation (day 1). During days $1-12$, we have repeated IP delivery of D1R agonist and observed a gradual decrease in the magnitude of the evoked signal over time. With a high PKI virus titer cohort, the D1R agonist response was eliminated by day 10 (Figures 3E,F). With a 10 -fold lower PKI virus titer cohort, the D1R agonist response was reduced to $30 \%$ of its amplitude on day 1 (Figures 3E,F). This reduction in FLIM-AKAR response is likely caused by the intended PKI effect of PKA inhibition as post hoc histological analysis showed minimal signs of neuronal death and confirmed expression of FLIM-AKAR.

Thus, given its dependence on PKA phosphorylation site and its blockade by PKI expression, we conclude that D1R-agonist induced decrease in FLIM-AKAR lifetime reflects bona fide activation of PKA in dSPNs. Furthermore, these results confirm the ability of FLiP to report dynamic modulation of endogenous biochemical signaling cascades in genetically-defined neurons in freely moving animals.

\section{FLiP Can Detect Modulation of PKA Activity in dSPNs and iSPNs Induced by a Natural Stimulus}

Although the D1R agonist experiment is a good proof-ofprinciple test for FLiP, the result of this experiment does not guarantee that the system will be able to pick up a change in PKA activity caused by a natural stimulus, which is likely to be smaller than the long-lasting effect of D1R agonism. Natural stimuli may induce signals that are more similar in kinetics and amplitude to the signals seen following IP saline injection (Figure 3C). Therefore, we tested if FLiP can detect changes in PKA activity in $\mathrm{dSPNs}$ and iSPNs in mice that receive a food reward. To compare PKA dynamics in dSPNSs and iSPNs, we expressed FLIM-AKAR in each population using Drd1a-Cre and Adora2aCre mice, respectively (Figure 4A). Two-three weeks after virus injection and fiber implantation, mice were food restricted for at least 1 day before the food reward response was measured. We subsequently measured the change in the fluorescence lifetime of FLIM-AKAR in these mice when we gave them a small food pellet $(20 \mathrm{mg}$ ) from a food receptacle in an otherwise empty chamber. Access to food in these mice was expected to elicit a dopamine transient in the NAc, resulting in subsequent activation $\mathrm{G}_{\alpha s}$-coupled dopamine receptors on dSPNs and $\mathrm{G}_{\alpha i}$-coupled dopamine receptors on iSPNs.

Indeed, FLIM-AKAR lifetime measured in Drd1a-Cre mice significantly decreased at the time of food consumption, as expected from an increase in dSPN PKA activity (Figure 4B).
A

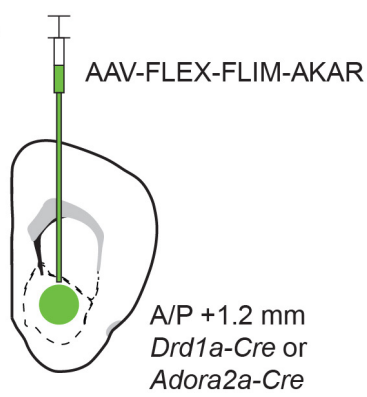

\section{B Receptacle Entry}

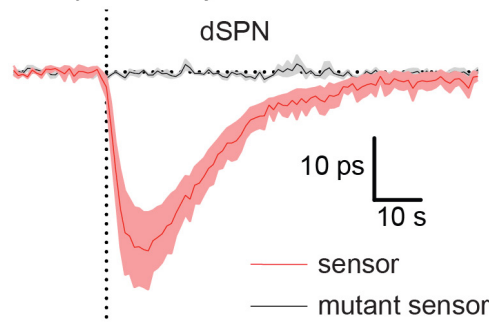

Receptacle Entry

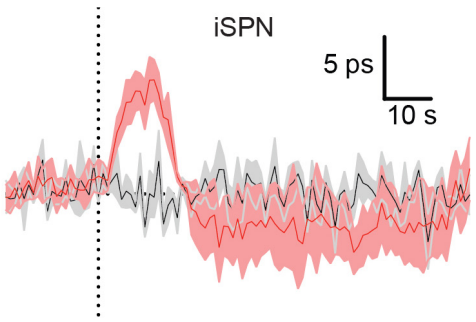

FIGURE 4 | Food rewards rapidly increase and decrease PKA phosphorylation in dSPNs and iSPNs, respectively. (A) Schematic of a coronal section at $1.2 \mathrm{~mm}$ depicting viral injection of AAV-FLEX-FLIM-AKAR into the NAc of a Drd1a-Cre or Adora2a-Cre mice. An optical fiber was implanted $200 \mu \mathrm{m}$ above the injection site. (B) Fluorescence lifetime changes of FLIM-AKAR (red) expressed in a dSPNs in Drd1-Cre mice (left) or iSPNs in Adora2a-Cre mice (right) when the animals were given a food reward (dashed vertical line = time point of food pellet receptacle entry). The mice were food restricted at least a day before the food reward response was measured. The patterns show an increase in PKA phosphorylation in dSPNs ( $n=4$ mice) and a decrease in iSPNs $(n=5$ mice). No change is seen in mice expressing FLIM-AKAR T391A (black) in either dSPNs $(n=3$ mice) or iSPNs $(n=4$ mice). Shaded area $=$ SEM of averages across mice. For each mouse, data were collected across 10 trials. 
Conversely, FLIM-AKAR lifetime in Adora2a-Cre mice increased at the time of food consumption, marking a reduction in PKA activity in iSPNs (Figure 4B). Neither signal was seen in mice expressing FLIM-AKAR ${ }^{T 391 A}$, confirming that the lifetime changes observed in FLIM-AKAR expressing animals on food consumption did not come from movement artifacts or changes in tissue autofluorescence.

The opposite changes in PKA activity in dSPNs and iSPNs are consistent with expected phasic dopamine release at the time of unexpected reward delivery leading to activation and suppression of PKA in each neuron class, respectively. Furthermore, these results demonstrate the utility of FLiP in monitoring changes in PKA activity in situ triggered by physiological stimuli in behaving animals.

\section{Expression of FLIM-AKAR Minimally Perturbs PKA Activity Dynamics}

A concern with reporters of physiological processes is that their expression may perturb the process of interest. For example, expression of a phosphorylation substrate could slow phosphorylation or dephosphorylation if kinases or phosphatases are in rate-limiting quantities. Such effects are typically seen as slowing of the signal as the levels of the reporters are increased. To examine if the expression of FLIM-AKAR perturbs the dynamics of PKA-dependent phosphorylation, we performed multi-day measurements of FLIM-AKAR lifetime changes in response to a food reward as the level of the reporter increased.

We injected AAV-FLEX-FLIM-AKAR into Drd1a-Cre mice (Figure 5A) and, after 3 days of surgery, started food restriction on the mice. Starting on the day after the beginning of food restriction, we measured FLIM-AKAR lifetime changes in response to a food reward across multiple days as the expression of FLIM-AKAR increased. The increase in FLIMAKAR expression was estimated from the basal level of fluorescence under constant excitation power (Figure 5B), which underestimates the true fold change in the reporter expression as it ignores that some fraction of the fluorescence arises from autofluorescence of the fiber and tissue. The daily average lifetime plot (averaged from five mice) showed no significant slowing of the dSPN PKA activity dynamics reported by FLIM-AKAR (Figures $\mathbf{5 C , D}$ ), even as the amplitude of the lifetime change increased over an $\sim 3$-fold. Furthermore, from day to day, there was no strong positive correlation between normalized photon counts and the time to maximal deviation from baseline ( $20-80 \%$ baseline to peak, $R^{2}=0.00078$ ), the time to return to baseline ( $80-20 \%$ peak to baseline, $R^{2}=0.12$ ), or the half-width of the signal $\left(R^{2}=0.012\right)$ (Figure 5D). Overall, we find no sign of limiting of PKA or phosphatase activity caused by the sensor expression. Of note, the fact that the fluorescence lifetime response increases between day $4 \sim$ day 10 and stabilizes after day 10 suggests that an accurate and stable measurement of fluorescence lifetime change requires a certain level of fluorophore concentration, which ensures the majority of the detected photons come from the sensor rather than the background (fiber and tissue autofluorescence).

\section{DISCUSSION}

Although many reporters of intracellular second messengers and their downstream signaling cascades have been developed, relatively little is known about the dynamic change of intracellular biochemical signaling other than calcium during behavior. Fluorescence lifetime imaging microscopy (FLIM), a FRET-based imaging approach, has been extensively used to study intracellular signaling in neurons ex vivo (Yasuda et al., 2006; Lee et al., 2009; Laviv et al., 2016; Chen et al., 2017; Tang and Yasuda, 2017). FLIM relies on detecting inter- or intra-molecular interactions between a donor and acceptor fluorophore through the FRET dependent change in the fluorescence lifetime of the donor. Genetically-encoded FLIM-compatible reporters have been developed for many kinases and second messengers (Yasuda et al., 2006; Lee et al., 2009; Klarenbeek et al., 2011; Chen et al., 2014; Laviv et al., 2016; Tang and Yasuda, 2017; Colgan et al., 2018; Ma et al., 2018). Inspired by these studies, we developed a single multimode fiber-based method to monitor intracellular signaling in freely-moving mice. We used fluorescence lifetime photometry (FLiP) to examine the dynamics of protein kinase A (PKA) signaling in specific population of neurons in the NAc. The PKA activity reporter that we used, FLIM-AKAR, has been heavily optimized for fluorescence lifetime measurements in the previous study (Chen et al., 2014). We demonstrate that FLiP, using a multimode fiber for both the fluorescence excitation and emission path, provides stable and reproducible measurements of the fluorescence lifetime of FLIM-AKAR. Furthermore, we were able to reveal modulation of the PKA phosphorylation state following pharmacological and behavioral stimuli, confirming the sensitivity of the approach to detect dynamics of intracellular biochemical signaling in behaving mice.

\section{Single Fiber FLiP}

The comparison of the lifetimes of a fluorescein standard measured by FLiP to those measured by 2P-FLIM shows that fiber photometry systematically underestimates lifetimes. We suggest that this is likely due to autofluorescence present in the fiber optic, which typically has a shorter lifetime $(\sim 2.7 \mathrm{~ns})$ than fluorescein ( $\sim 4.1 \mathrm{~ns}$ ). This conclusion is supported by the finding that adding the brain fiber implant to the end of the patch cord lowers the measured lifetime further, likely reflecting the additional fluorescence derived from this device. Nevertheless, utilizing a single multimode fiber for excitation light delivery to the brain and collection of fluorescence from the brain, we were able to measure a biologically meaningful fluorescence lifetime change.

Our results demonstrate that single-fiber FLiP is able to detect pharmacologically- and behaviorally-induced changes in PKAdependent phosphorylation in genetically-defined populations of neurons in the NAc. Based on the results presented above, we propose that the relatively simple single-fiber system presented here provides a powerful method for the analysis of intracellular signaling cascades in behaving animals.

\section{Comparison to Other Approaches}

Real-time reporters of biochemical processes typically rely on intra- or inter-molecular conformational changes induced by 
A

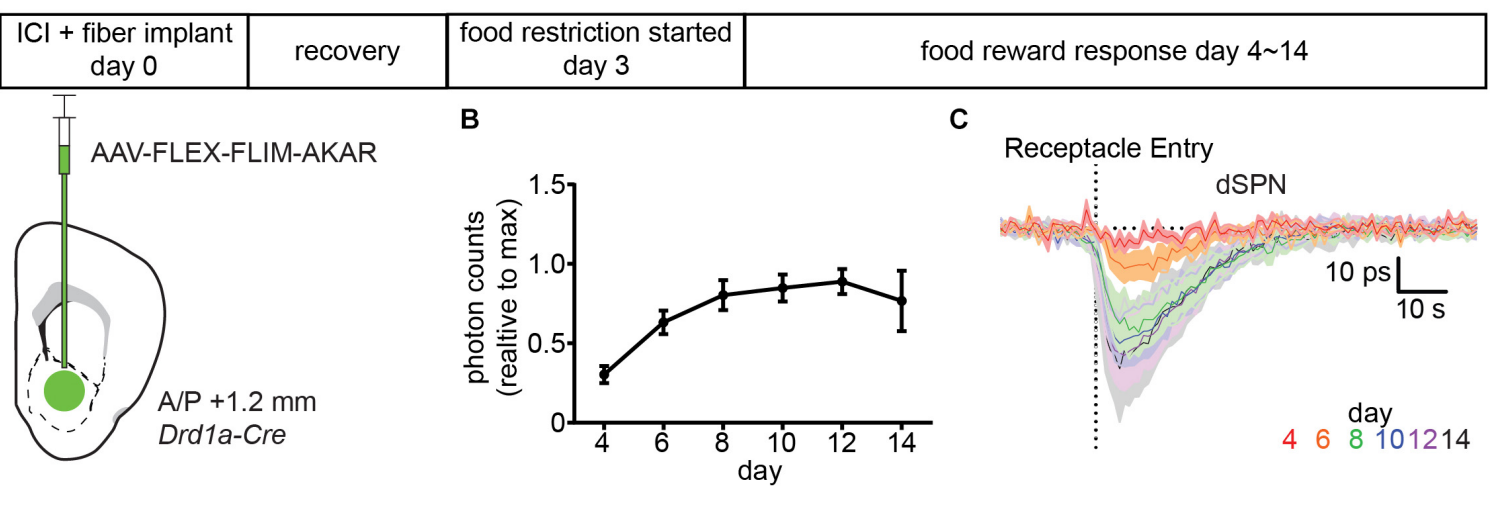

D
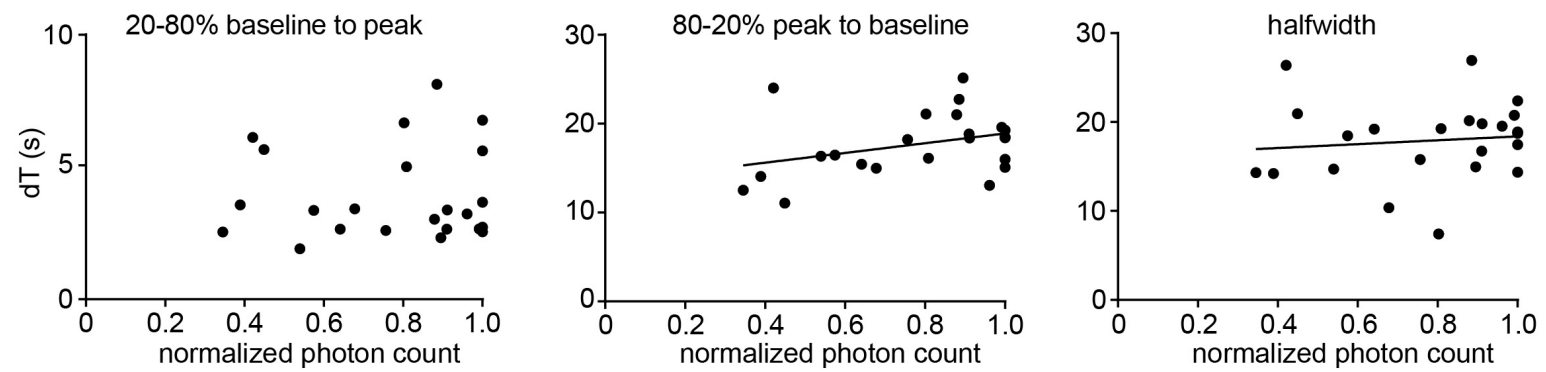

FIGURE 5 | Expression of FLIM-AKAR minimally perturbs PKA activity dynamics. (A) Schematic of the experimental design for testing the effects of FLIM-AKAR expression levels on response kinetics. First, a Drd1a-Cre mouse was injected with AAV-FLEX-FLIM-AKAR intracranially and was implanted with a fiber. After 3 days of recovery, the mouse was started on food restriction. Starting on day 4 after surgery (1 day after the beginning of food restriction), food induced changes in lifetime of FLIM-AKAR expressed in dSPNs were measured for 10 days. After 10 days, the fluorescence intensity reached its maximum for all mice, suggesting viral sensor expression had reached its maximum (B). (B) Average photon counts of FLIM-AKAR fluorescence under the fixed excitation power across multiple days, normalized for each mouse by its maximum photon count. Error bar $=$ SEM across $n=5$ mice. (C). Average food-induced change in lifetime of FLIM-AKAR expressed in dSPNs of Drd1a-Cre mice across days. The dashed vertical line indicates the time when the animals entered the food pellet receptacle. Shaded area $=$ SEM across $n=5$ mice. For each mouse, data were collected across 6-10 trials/day. (D) For each mouse on each day, the FLIM-AKAR response kinetics were quantified by measuring the time required for the signal to reach $80 \%$ of the maximum valley amplitude from $20 \%$ of the maximum valley amplitude (20-80\% baseline to peak) (left) and the time to return to $20 \%$ of the maximum valley amplitude from $80 \%$ of the maximum valley amplitude (80-20\% peak to baseline) (middle). In addition, the halfwidth (right) of the FLIM-AKAR lifetime response was measured. Each parameter is plotted as a function of the baseline fluorescence photon counts measured in each mouse for each day during periods when the animal was not eating. $N=5$ mice with $6-10$ trials acquired for each data point. Measurements were analyzed when the fluorescence intensity reached $30 \%$ of its maximum as they were too noisy at lower intensities.

an enzymatic modification, such as phosphorylation, or binding of a bioactive molecule. Some reporters, such as GCaMP, alter their brightness on activation due to a change in absorption cross-section or quantum yield, which typically does not alter the fluorescence lifetime of the fluorophore. These reporters are usually monitored using a single excitation and emission wavelength. When these reporters are used with fiber photometry to collect bulk signals in moving animals, care is needed to ensure that fluorescence dynamics reflect the target biological processes, as opposed to fluorescence changes induced by movement of the optical fiber, changes in $\mathrm{pH}$ in the tissue, or hemodynamic alterations that affect photon absorption and scattering (Hires et al., 2008; Cui et al., 2013; Valley et al., 2019).

On the other hand, with two-color FRET sensors, the fluorescence intensities of the acceptor and the donor are both monitored, and their ratio is calculated to deduce changes in biochemical states. Such approach is difficult to implement in vivo, as many factors, such as differential bleaching of each fluorophore, overlap in the emission or excitation spectra, differential sensitivity of each spectrum to hemodynamic changes, and differential contribution of autofluorescence to each emission spectrum, will alter the ratio of donor and acceptor fluorescence intensity.

For FRET-based FLIM reporters, activation of the biochemical process alters the fluorescence lifetime of the donor fluorophore, which allows their monitoring by FLiP and other lifetime detection methods. Single fluorophore FLIM method avoids many of the complications of two-color FRET method mentioned above as the FLIM measurement - fluorescence lifetime of the donor - is in principal independent of the concentration of the donor molecules, the excitation efficiency that depends on change in illumination, and the efficiency with which the fluorescence is collected. In practice, this holds true as long as the contribution of background fluorescence to the detected signal is minimal compared to that of the sensor (the majority of the detected photons come from the donor fluorophore).

Fluorescence lifetime imaging microscope and, by extension, FLiP are not impervious to artifacts, and controls for the specificity of observed lifetime transients need to be established as they were done here using a variety of controls. Of particular 
concern is the non-negligible contribution of autofluorescence from the optical fiber, which can affect the measured fluorescence lifetime if the reporter is dim or rapidly bleaching, compared to the autofluorescence. In our experience, this concern can be avoided by using low excitation light levels that minimize reporter bleaching. Nevertheless, because of the contribution of autofluorescence, we avoid translating absolute lifetime measurements into ratios of active and inactive reporter, as it is often done with low-background imaging modalities such as $2 \mathrm{P}-$ FLIM.

\section{PKA Dynamics in the NAc}

We find that pharmacological activation of Type 1 dopamine receptors (D1Rs) using peripheral administration of an agonist induces robust and long-lived (>20 min) increases in PKA dependent phosphorylation in D1R-expressing dSPNs of the NAc. This is consistent with the GPCR biology of D1Rs: they couple to Gas to increase cAMP concentration and thus activate PKA. The dependence of the reporter response on the PKA phosphorylation site of the reporter and its blockade by expression of PKI, demonstrate that the reporter response results from PKA phosphorylation of FLIM-AKAR. As D1Rs couple through $\mathrm{G}_{\alpha s}$ to increase cAMP concentration and thus activate PKA, this was the expected result. In addition to confirming the sensitivity of fiber-based measurements of fluorescence lifetime in vivo, these data reveal the in vivo pharmacokinetics of SKF 81297 hydrobromide, which is widely used in behavioral experiments. Because FLIM-AKAR can report orders-of-magnitude briefer ( $\sim 20 \mathrm{~s})$ behaviorally triggered PKA transients (see below), the kinetics of PKA activation reported by the sensor following D1R agonism likely reflect the duration of exposure of NAc dSPNs to the drug. We propose that analysis of biochemical state using in vivo FLiP can be an important tool to determine the brain penetrance, exposure time, or mechanism of action of drugs targeting the central nervous system.

Delivery of a reward, in the form of a food pellet to foodrestricted animals, transiently increases and decreases PKA activity in dSPNs and iSPNs in the NAc, respectively. These opposite changes are predicted based on the expression of $\mathrm{G}_{\alpha s^{-}}$ and $\mathrm{G}_{\alpha i}$-coupled receptors in $\mathrm{dSPNs}$ and iSPNs, respectively. As the kinetics of these signals are not artificially prolonged by the level of reporter expression, they provide an estimate of the kinetics of combined PKA/phosphatase action on diffusible PKA substrates in the cell. Thus, food reward induced changes in PKA dependent phosphorylation in dSPNs and iSPNs, for $\sim 40$ and $\sim 20 \mathrm{~s}$, respectively, which provides an upper estimate of the kinetics of the intracellular biochemical change. In addition, we find that rewards lower phosphorylation state in iSPNs, confirming that, in these cells, there is a basal phosphorylation of PKA substrates, which can be reversed by inhibition of adenylyl cyclases via $\mathrm{G}_{\alpha i}$-coupled GPCRs such as Type 2 dopamine receptors.

\section{Summary and Outlook}

The fluorescence lifetime photometry system described here is relatively simple to implement. As it is based on a single multimode fiber, it is compatible with low cost launches that couple the laser into the fiber. Future improvements may include replacing the expensive time correlated single photon counting boards with more affordable high-speed field-programmable gate arrays (FPGAs) that are more flexible and do not suffer from dead time, which can increase sampling rates. The system presented here can detect behaviorally and pharmacologically induced transients in intracellular biochemical state in genetically-defined neurons. Thus, it will permit further studies of the intracellular biochemical mechanisms of many brain processes, such as those linking experience to cellular plasticity, mediating activity dependent circuit regulation, and by which hormonal and metabolic signals influence cellular states.

\section{MATERIALS AND METHODS}

Experimental manipulations were performed in accordance with protocols approved by the Harvard Standing Committee on Animal Care following guidelines described in the US National Institutes of Health Guide for the Care and Use of Laboratory Animals. Inhaled isoflurane was used as anesthesia.

\section{2-Photon Fluorescence Lifetime Imaging Microscopy (2P-FLIM)}

2-Photon fluorescence lifetime imaging microscopy (Figure 2) was carried out using a custom microscope as previously described (Chen et al., 2014). $920 \mathrm{~nm}$ excitation light (Chameleon Vision II, $80 \mathrm{MHz}$, Coherent, Santa Clara, CA, United States) was used to 2-photon excite fluorescein dissolved in water, and resulted fluorescence was collected in the 500-550 $\mathrm{nm}$ bank using a 525/25 nm emission filter (Semrock) and a PMT (H742240 MOD Hamamatsu).

\section{Fluorescence Lifetime Photometry (FLiP)}

Fluorescence lifetime photometry was carried out as illustrated in Figure 1. All filters in the system were made by Semrock. The 473 nm laser BDS-473-SM-FBE [Becker and Hickl (BH)] operated at $50 \mathrm{Mhz}$ was used as a pulsed light source. For detection, a high speed hybrid PMT, HPM-100-07-Cooled (BH) controlled by DCC-100-PCI (BH) was used. The PMT was connected to SPC-830 (BH), a time correlated single photon counting (TCSPC) board, which detects the time delay between the pulsed excitation and the photon detection by the PMT. The data was collected by a custom software in MATLAB, which calculated the average lifetime of detected photons at 1 $s$ intervals. This interval for average lifetime measurements was empirically determined to have enough photons to accurately estimate the lifetime ( $>200,000$ photons/measurement) without running into a photon count limit $(<1,000,000$ photons/s) of the TCSPC board. The typical excitation power needed to generate an appropriate amount of photons for TCSPC was $0.6 \sim 1 \mu \mathrm{W}$ (measured at the output end of the patch cord).

For the estimation of the absolute lifetime of a fluorescein solution standard (Figure 2), we fit a single exponential decay to the lifetime histogram. For the data presented in Figure 2, we used the time constant of this single exponential decay as the 
measure of lifetime. For the estimation of the change in lifetime of the sensor in vivo (Figures 3-5), we calculated, for each $1 \mathrm{~s}$ time bin, an average lifetime by measuring the mean photon arrival time (the population mean of the delay between the pulsed excitation and the fluorescence photon arrival as described in the equation below), as discussed in the previous study by Lee et al. (2009).

$$
\tau_{m} \mid=<t>-t_{0}=\frac{\int d t \cdot t F(t)}{d t \cdot F(t)}-t_{0}
$$

Here, $\mathrm{F}(\mathrm{t})$ is the photon count of a fluorescence lifetime decay curve at time bin $t$, and $t_{0}$ is the offset of the lifetime histogram, which can be estimated by fitting a double exponential curve to a lifetime histogram once in the beginning of the experiment.

For this calculation, we chose a time range (0-8 ns) in a lifetime histogram that was minimally contaminated by a secondary fluorescence peak created by the autofluorescence of a fiber. The length of the patch cord was chosen to maximize the time separation between the sensor fluorescence peak and the fiber autofluorescence peak ( $\sim 10$ ns time delay for light to travel from the one end of the patch cord to the other end and back).

The lifetime of each fluorophore was reported as a change in lifetime (delta lifetime), which was calculated by subtracting the average lifetime of a baseline period (a period in graphs before the event of interest) from the average lifetime transient.

\section{Surgery}

Drd1a-Cre or Adora2a-Cre mice with C57BL/6J background from MMRRC UC Davis were used for all in vivo experiments. AAV1-FLEX-FLIM-AKAR $\left(2.14 \times 10^{13} \mathrm{gc} / \mathrm{ml}, 300 \mathrm{nl}\right)$, AAV1FLEX-FLIM- FLIM-AKAR ${ }^{\text {T391A }}\left(7.67 \times 10^{12} \mathrm{gc} / \mathrm{ml}, 300 \mathrm{nl}\right)$, AAV-FLEX-PKIalpha-IRES-nls-mRuby2 $\left(3.72 \times 10^{13} \mathrm{gc} / \mathrm{ml}\right.$ for low titer and $3.72 \times 10^{14} \mathrm{gc} / \mathrm{ml}$ for high titer, $\left.600 \mathrm{nl}\right)$ were stereotactically injected at $+1.2 \mathrm{~mm}(\mathrm{~A} / \mathrm{P}),+1.3 \mathrm{~mm}(\mathrm{M} / \mathrm{L})$ from bregma at a depth of $4.1 \mathrm{~mm}$ from brain surface. A fiber optic implant (MFC_200/230-0.37_4.5mm_MF1.25_FLT mono fiber optic cannula or DFC_200/245-0.37_5.5mm_DF0.7(2.5)_FLT dual fiber optic cannula from Doric Lenses) was implanted at the same coordinate at a depth of $3.9 \mathrm{~mm}$ from the brain surface. The dual fiber optic implant was custom made to place the two fibers adjacent to each other without any gap and thus to maximize the overlap between the excitation and collection volumes.

\section{REFERENCES}

Beninger, R. J., and Gerdjikov, T. V. (2004). The role of signaling molecules in reward-related incentive learning. Neurotox. Res. 6, 91-103. doi: 10.1007/ BF03033301

Berke, J. D., and Hyman, S. E. (2000). Addiction, Dopamine, and the molecular mechanisms of memory. Neuron 25, 515-532. doi: 10.1016/S0896-6273(00) 81056-9

Bromberg-Martin, E. S., Matsumoto, M., and Hikosaka, O. (2010). Dopamine in motivational control: rewarding, aversive, and alerting. Neuron 68, 815-834. doi: 10.1016/j.neuron.2010.11.022

Carvalho, A. L., Kameyama, K., and Huganir, R. L. (1999). Characterization of phosphorylation sites on the glutamate receptor 4 subunit of the AMPA receptors. J. Neurosci. 19, 4748-4754. doi: 10.1016/S0896-6273(00)8 0144-0

\section{Pharmacology}

SKF 81297 hydrobromide $(10 \mathrm{mg} / \mathrm{kg}$ mixed in $0.1 \mathrm{ml}$ of saline/10 $\mathrm{g}$ of mouse) was injected intraperitoneally (IP) to activate Type 1 dopamine receptors (D1R) (Figure 3). Saline (0.1 $\mathrm{ml}$ of saline/10 $\mathrm{g}$ of mouse) was injected IP for the controls trials.

\section{Behavior}

A mouse connected to a patch cord was allowed to freely move in an $8 \times 16$ inch behavior box, which contained a pellet receptacle in one wall. Receptacle entry was detected by an infrared sensor installed inside the receptacle. The time of beam break was used to align the fluorescence lifetime measurements across trials and to analyze changes in lifetime caused by food rewards.

\section{DATA AVAILABILITY}

The datasets generated for this study are available on request to the corresponding author.

\section{ETHICS STATEMENT}

The animal study was reviewed and approved by Harvard Medical School IACUC.

\section{AUTHOR CONTRIBUTIONS}

BS conceived, analyzed, and wrote the manuscript. SL collected the data, conceived, analyzed, and wrote the manuscript. YC conceived and edited the manuscript. BL collected the data and analyzed the manuscript.

\section{FUNDING}

This work was funded by NIH (NINDS R35NS105107, BS), Sackler Fellowship (SL), and Howard Hughes Medical Institute.

Chen, Y., Granger, A. J., Tran, T., Saulnier, J. L., Kirkwood, A., and Sabatini, B. L. (2017). Endogenous Gaq-coupled neuromodulator receptors activate protein kinase A. Neuron 96, 1070-1083.e5. doi: 10.1016/j.neuron.2017.10.023

Chen, Y., Saulnier, J. L., Yellen, G., and Sabatini, B. L. (2014). A PKA activity sensor for quantitative analysis of endogenous GPCR signaling via 2-photon FRET-FLIM imaging. Front. Pharmacol. 5:56. doi: 10.3389/fphar.2014.00056

Colgan, L. A., Hu, M., Misler, J. A., Parra-bueno, P., Moran, C. M., Leitges, M., et al. (2018). PKC $\alpha$ integrates spatiotemporally distinct $\mathrm{Ca} 2+$ and autocrine BDNF signaling to facilitate synaptic plasticity. Nat. Neurosci. 21, 1027-1037. doi: 10.1038/s41593-018-0184-3

Cui, G., Jun, S. B., Jin, X., Luo, G., Pham, M. D., Lovinger, D. M., et al. (2014). Deep brain optical measurements of cell type-specific neural activity in behaving mice. Nat. Protoc. 9, 1213-1228. doi: 10.1038/nprot.2014.080

Cui, G., Jun, S. B., Jin, X., Pham, M. D., Vogel, S. S., Lovinger, D. M., et al. (2013). Concurrent activation of striatal direct and indirect 
pathways during action initiation. Nature 494, 238-242. doi: 10.1038/ nature 11846

Dalton, G. D., and Dewey, W. L. (2006). Protein kinase inhibitor peptide (PKI): a family of endogenous neuropeptides that modulate neuronal cAMP-dependent protein kinase function. Neuropeptides 40, 23-34. doi: 10.1016/j.npep.2005. 10.002

Davis, R. L. (1996). Physiology and biochemistry of Drosophila learning mutants. Physiol. Rev. 76, 299-317. doi: 10.1152/physrev.1996.76.2.299

Doll, C. A., and Broadie, K. (2014). Impaired activity-dependent neural circuit assembly and refinement in autism spectrum disorder genetic models. Front. Cell. Neurosci. 8:30. doi: 10.3389/fncel.2014.00030

Gerfen, C. R., and Surmeier, D. J. (2011). Modulation of striatal projection systems by dopamine. Annu. Rev. Neurosci. 34, 441-466. doi: 10.1146/annurev-neuro061010-113641

Goto, A., Nakahara, I., Yamaguchi, T., Kamioka, Y., Sumiyama, K., Matsuda, M., et al. (2015). Circuit-dependent striatal PKA and ERK signaling underlies rapid behavioral shift in mating reaction of male mice. Proc. Natl. Acad. Sci. U.S.A. 112, 6718-6723. doi: 10.1073/pnas.1507121112

Greengard, P. (2001). The neurobiology of slow synaptic transmission. Science 294, 1024-1030. doi: 10.1126/science.294.5544.1024

Herring, B. E., and Nicoll, R. A. (2016). Long-term potentiation: from CaMKII to AMPA receptor trafficking. Annu. Rev. Physiol. 78, 351-365. doi: 10.1146/ annurev-physiol-021014-071753

Hires, S. A., Tian, L., and Looger, L. L. (2008). Reporting neural activity with genetically encoded calcium indicators. Brain Cell Biol. 36, 69-86. doi: 10.1007/ s11068-008-9029-4

Howe, M. W., and Dombeck, D. A. (2016). Rapid signalling in distinct dopaminergic axons during locomotion and reward. Nature 535, 505-510. doi: $10.1038 /$ nature 18942

Huang, E. J., and Reichardt, L. F. (2001). NEUROTROPHINS: roles in neuronal development and function. Annu. Rev. Neurosci. 24, 677-736. doi: 10.1146/ annurev.neuro.24.1.677

Jiang, X., and Nardelli, J. (2016). Neurobiology of disease cellular and molecular introduction to brain development. Neurobiol. Dis. 92, 3-17. doi: 10.1016/j.nbd. 2015.07.007

June, A. J. M. (2012). Pharmacological PKA inhibition: all may not be what it seems. Sci. Signal. 1:re4. doi: 10.1126/scisignal.122re4

Kandel, E., and Abel, T. (1995). Neuropeptides, adenylyl cyclase, and memory storage. Science 268, 825-826. doi: 10.1126/science.7754367

Kandel, E. R. (2012). The molecular biology of memory cAMP, PKA, CRE, CREB-1, CREB-2, and CPEB. Mol. Brain 5:14. doi: 10.1186/1756-6606-5-14

Klarenbeek, J. B., Goedhart, J., Hink, M. A., Gadella, T. W. J., and Jalink, K. (2011). A mTurquoise-based cAMP sensor for both FLIM and ratiometric read-out has improved dynamic range. PLoS One 6:e19170. doi: 10.1371/journal.pone. 0019170

Kravitz, A. V., and Kreitzer, A. C. (2012). Striatal mechanisms underlying movement, reinforcement, and punishment. Physiology 27, 167-177. doi: 10 . 1152/physiol.00004.2012

Kreitzer, A. C., and Malenka, R. C. (2008). Striatal plasticity and basal ganglia circuit function. Neuron 60, 543-554. doi: 10.1016/j.neuron.2008.11.005

Lau, G. C., Saha, S., Faris, R., and Russek, S. J. (2004). Up-regulation of NMDAR1 subunit gene expression in cortical neurons via a PKA-dependent pathway. J. Neurochem. 88, 564-575. doi: 10.1046/j.1471-4159.2003.02156.x

Laviv, T., Kim, B. B., Chu, J., Lam, A. J., Lin, M. Z., and Yasuda, R. (2016). Simultaneous dual-color fluorescence lifetime imaging with novel red-shifted fluorescent proteins. Nat. Methods 13, 989-992. doi: 10.1038/nmeth.4046

Lee, S. R., Escobedo-lozoya, Y., Szatmari, E. M., and Yasuda, R. (2009). Activation of CaMKII in single dendritic spines during long-term potentiation. Nature 458, 299-304. doi: 10.1038/nature07842

Ma, L., Jongbloets, B. C., Xiong, W., Zemelman, B. V., Mao, T., Ma, L., et al. (2018). A highly sensitive A-Kinase activity reporter for imaging neuromodulatory events in awake mice. Neuron 99, 665.e-679.e. doi: 10.1016/j.neuron.2018. 07.020

Nayak, A., Zastrow, D. J., Lickteig, R., Zahnister, N. R., and Browning, M. D. (1998). Maintenance of late-phase LTP is accompanied by PKA-dependent increase in AMPA receptor synthesis. Nature 396, 482-486. doi: 10.1038/nature01665.1

Prieto, G. A., and Cotman, C. W. (2017). Cytokine \& growth factor reviews cytokines and cytokine networks target neurons to modulate long-term potentiation. Cytokine Growth Factor. Rev. 34, 27-33. doi: 10.1016/j.cytogfr. 2017.03.005

Regehr, W. G., Carey, M. R., and Best, A. R. (2009). Review activity-dependent regulation of synapses by retrograde messengers. Neuron 63, 154-170. doi: 10.1016/j.neuron.2009.06.021

Schultz, W. (2013). Updating dopamine reward signals wolfram schultz. Curr. Opin. Neurobiol. 23, 229-238. doi: 10.1016/j.conb.2012.11.012

Schultz, W., Dayan, P., and Montague, P. R. (1997). A neural substrate of prediction and reward. Science 275, 1593-1600. doi: 10.1126/science.275.5306. 1593

Shaywitz, A. J., and Greenberg, M. E. (1999). CREB: a stimulus-induced transcription factor activated by a diverse array of extracellular signals. Annu. Rev. Biochem. 68, 821-861. doi: 10.1146/annurev.biochem.68.1.821

Shen, W., Flajolet, M., Greengard, P., and Surmeier, D. J. (2008). Dichotomous control dopaminergic of striatal synaptic plasticity. Science 321, 848-851. doi: 10.1126/science.1160575.Dichotomous

Skeberdis, V. A., Chevaleyre, V., Lau, C. G., Goldberg, J. H., Pettit, D. L., Suadicani, S. O., et al. (2006). Protein kinase A regulates calcium permeability of NMDA receptors. Nat. Neurosci. 9, 501-510. doi: 10.1038/nn1664

Tang, S., and Yasuda, R. (2017). Imaging ERK and PKA activation in single dendritic spines during structural plasticity. Neuron 93, 1315-1324.e3. doi: 10.1016/j.neuron.2017.02.032

Tritsch, N. X., and Sabatini, B. L. (2012). Dopaminergic modulation of synaptic transmission in cortex and striatum. Neuron 76, 33-50. doi: 10.1016/j.neuron. 2012.09.023

Tronson, N. C., Wiseman, S. L., Olausson, P., and Taylor, J. R. (2006). Bidirectional behavioral plasticity of memory reconsolidation depends on amygdalar protein kinase A. Nat. Neurosci. 9, 167-169. doi: 10.1038/nn1628

Valley, M. T., Moore, M. G., Zhuang, J., Mesa, N., Castelli, D., Sullivan, D., et al. (2019). Separation of hemodynamic signals from GCaMP fluorescence measured with widefield imaging. bioRxiv [Preprint]. doi: 10.1101/634923

Yagishita, S., Hayashi-Takagi, A., Ellis-Davies, G. C. R., Urakubo, H., and Ishii, S. (2014). A critical time window for dopamine actions on the structural plasticity of dendritic spines. Science 345, 1616-1621. doi: 10.1126/science.12 55514

Yamaguchi, T., Goto, A., Nakahara, I., Yawata, S., Hikida, T., Matsuda, M., et al. (2015). Role of PKA signaling in D2 receptor-expressing neurons in the core of the nucleus accumbens in aversive learning. Proc. Natl. Acad. Sci. U.S.A. 112, 11383-11388. doi: 10.1073/pnas.1514731112

Yasuda, R., Harvey, C. D., Zhong, H., Sobczyk, A., Van Aelst, L., and Svoboda, K. (2006). Supersensitive Ras activation in dendrites and spines revealed by twophoton fluorescence lifetime imaging. Nat. Neurosci. 9, 283-291. doi: 10.1038/ nn 1635

Conflict of Interest Statement: The authors declare that the research was conducted in the absence of any commercial or financial relationships that could be construed as a potential conflict of interest.

Copyright (c) 2019 Lee, Chen, Lodder and Sabatini. This is an open-access article distributed under the terms of the Creative Commons Attribution License (CC BY). The use, distribution or reproduction in other forums is permitted, provided the original author(s) and the copyright owner(s) are credited and that the original publication in this journal is cited, in accordance with accepted academic practice. No use, distribution or reproduction is permitted which does not comply with these terms. 